\title{
Fast dispersive beam deflectors and modulators
}

\author{
Filinski, Ignacy; Skettrup, Torben
}

Published in:

I E E E Journal of Quantum Electronics

Link to article, DOI:

10.1109/JQE.1982.1071657

Publication date:

1982

Document Version

Publisher's PDF, also known as Version of record

Link back to DTU Orbit

Citation (APA):

Filinski, I., \& Skettrup, T. (1982). Fast dispersive beam deflectors and modulators. I E E E Journal of Quantum Electronics, 18(7), 1059-1062. https://doi.org/10.1109/JQE.1982.1071657

\section{General rights}

Copyright and moral rights for the publications made accessible in the public portal are retained by the authors and/or other copyright owners and it is a condition of accessing publications that users recognise and abide by the legal requirements associated with these rights.

- Users may download and print one copy of any publication from the public portal for the purpose of private study or research.

- You may not further distribute the material or use it for any profit-making activity or commercial gain

- You may freely distribute the URL identifying the publication in the public portal

If you believe that this document breaches copyright please contact us providing details, and we will remove access to the work immediately and investigate your claim. 


\section{ACKNOWLEDGMENT}

The authors wish to thank T. Shiono for his helpful assistance in the experimental work.

\section{REFERENCES}

[1] D. B. Anderson, J. T. Boyd, M. C. Hamilton, and R. R. August, "An integrated-optical approach to the Fourier transform," IEEE J. Quantum Electron., vol. QE-13, pp. 268-275, Apr. 1977.

[2] M. K. Barnoski, B. U. Chen, T. R. Joseph, J. Y. Lee, and O. G. Ramer, "Integrated-optic spectrum analyzer," IEEE Trans. Circuits Syst., vol. CAS-26, pp. 1113-1124, Dec. 1979.

[3] D. Mergerian, E. C. Malarkey, R. P. Pautienus, J. C. Bradley, G. E. Marx, L. D. Hutcheson, and A. L. Kellner, "Operational integrated optical RF spectrum analyzer," Appl. Opt., vol. 19, pp. 3033-3034, Sept. 1980.

[4] B. Chen, E. Marom, and A. Lee, "Geodesic lenses in single-mode. $\mathrm{LiNbO}_{3}$ waveguides," Appl. Phys. Lett., vol. 31, pp. 263-265, Aug. 1977.

[5] S. K. Yao and D. B. Anderson, "Shadow sputtered diffractionlimited waveguide Luneburg lenses," Appl. Phys. Lett., vol. 33, pp. 307-309, Aug. 1978.

[6] S. K. Yao and D. E. Thompson, "Chirp-grating lens for guidedwave optics," Appl. Phys. Lett, vol. 33, pp. 635-637, Oct. 1978.
[7] W.S.C. Chang and P. R. Ashley, "Fresnel lenses in optical waveguides," IEEE J. Quantum Electron., vol. QE-16, pp. 744-754, July 1980.

[8] V. Neuman, C. W. Pitt, and L. M. Walpita, "An integrated acoustooptic spectrum analyzer using grating components," in Proc. 1 st European Conf. Integrated Optics, London, England, Sept. 14-15, 1981, pp. 89-92.

[9] C. S. Tsai, "Guided-wave acoustooptic Bragg modulators for wideband integrated optic communications and signal processing," IEEE Trans. Circuits Syst., vol. CAS-26, pp. 1072-1098, Dec. 1979.

[10] T. Suhara, H. Nishihara, J. Koyama, J. Viljanen, and M. Leppihalme, "Integrated-optic wavelength multi- and demultiplexers using a chirped grating and an ion-exchanged waveguide," Proc. 1st European Conf. Integrated Optics, London, England, Sept. 14-15, 1981, post-deadline paper, pp. 12-14.

[11] T. Suhara, J. Viljanen, and M. Leppihalme, "Integrated-optic wavelength multi- and demultiplexers using a chirped grating and an ion-exchanged waveguide," Appl. Opt., vol. 21, May 1982 , to be published.

[12] Y. Handa, T. Suhara, H. Nishihara, and J. Koyama, "Microgratings for high-efficiency guided-beam deflection fabricated by electron-beam direct-writing techniques," Appl. Opt., vol. 19, pp. 2842-2847, Aug. 1980.

[13] H. Nishihara, Y. Handa, T. Suhara, and J. Koyama, "Electronbeam directly written microgratings for integrated optical circuits," Proc. SPIE, vol. 239, pp. 134-141, 1980.

\title{
Fast Dispersive Beam Deflectors and Modulators
}

\author{
I. FILINSKI AND T. SKETTRUP
}

\begin{abstract}
A new method for one-dimensional light scanning is proposed. It is based on the use of ordinary dispersive optical components like prisms, gratings, etc. By electrooptic tuning of the output wavelength of broad-band lasers, fast scanners (up to 10 gigapixels/s) can be constructed. Deflection angles and numbers of pixels are derived for the prism, grating, Fabry-Perot etalon, and phase-matched frequency doubler crystal. Experimental results on the frequency doubler device are presented.
\end{abstract}

\section{INTRODUCTION}

T HE existence of broad-band tunable lasers such as dye lasers or the alexandrite laser implies that it is possible to construct fast line scanners or modulators by means of a dispersive element and a tuning element activated by electric or magnetic fields.

Telle and Tang [1] demonstrated an electrooptic tuning element in 1974. Later, a similar device became commercially available with tuning rates up to $80 \mathrm{~nm} / \mathrm{kV}( \pm 7,5 \mathrm{kV})$ and maximum frequencies up to $5 \mathrm{MHz}$ [2]. Using such a tuning element in a dye laser or an alexandrite laser together with a dispersive element it is possible to obtain scanning rates up to the order of $10^{10}$ pixels $/ \mathrm{s}$ as discussed below. The scanning

Manuscript received March 23, 1982. This work was supported by the Danish Natural Science Research Council.

The authors are with the Physics Laboratory III, Technical University of Denmark, Lyngby, Denmark. takes place in one dimension. Another type of deflector must be added in order to obtain two-dimensional scanning.

In the following we shall discuss four dispersive components which can be applied as deflectors or modulators: the prism, the grating, the Fabry-Perot etalon, and the phase-matched frequency doubler. Three quantities are important when specifying these kinds of deflectors or modulators, the angular deflection per unit wavelength $d \theta / d \lambda$, the number of pixels (picture elements) per unit wavelength $d N / d \lambda$, and the total number of pixels $N$ obtainable with the system. From these it is easy to find the angular deflection $\Delta \theta$ and the wavelength increase $\Delta \lambda$ per pixel which are more relevant for modulators.

The resolving power $A$ of a dispersive element is given by

$$
A=\frac{\lambda}{\Delta \lambda}
$$

where $\Delta \lambda$ is the wavelength interval just resolved by the element. Hence, the wavelength increase per pixel is

$$
\Delta \lambda=\frac{\lambda}{A}
$$

and the number of pixels per unit wavelength is

$$
\frac{d N}{d \lambda}=\frac{1}{\Delta \lambda}=\frac{A}{\lambda} .
$$


The total number of pixels for the system is then

$$
N=\frac{d N}{d \lambda}\left(\lambda_{2}-\lambda_{1}\right)
$$

where $\lambda_{1}$ and $\lambda_{2}$ are the tuning limits of the laser.

A prism has an angular deflection per unit wavelength given by [3]

$$
\frac{d \theta}{d \lambda}=\frac{b}{h} \frac{d n}{d \lambda}
$$

where $b$ and $h$ are base and height of the prism and $n$ is the refractive index. The resolution of a prism is given by [4]

$$
A=b \frac{d n}{d \lambda}
$$

if the prism is completely filled with light. Hence, according to (3)

$$
\frac{d N}{d \lambda}=\frac{b}{\lambda} \frac{d n}{d \lambda} .
$$

A grating has an angular deflection per unit wavelength given by [5]

$$
\frac{d \theta}{d \lambda}=\left(\left(\frac{d}{m}\right)^{2}-\left(\lambda+\left(\frac{d}{m}\right) \sin \theta_{0}\right)^{2}\right)^{-1 / 2}
$$

where $d$ is the spacing between the grooves, $m$ is the order, and $\theta_{0}$ is the angle of incidence. The resolution of the grating is [5]

$$
A=m \frac{D}{d}
$$

where $D$ is the diameter of the laser beam at the grating, implying

$$
\frac{d N}{d \lambda}=\frac{m}{d} \frac{D}{\lambda} .
$$

A Fabry-Perot etalon has an angular deflection per unit wavelength given by [6]

$$
\frac{d \theta}{d \lambda}=-\frac{\cot \theta}{\lambda}
$$

which increases strongly for fringes close to the axis. Close to $\theta=0$ (11) is not useful. Here the variation in $\theta$ required to move a passband by one order is approximately [6]

$$
\Delta \theta \simeq\left(\frac{\lambda}{n d}\right)^{1 / 2}
$$

where $n d$ is the optical length of the etalon. The corresponding wavelength change (free spectral range) is

$$
\Delta \lambda=\frac{\lambda^{2}}{2 n d}
$$

yielding an approximate deflection rate close to $\theta=0$

$$
\frac{d \theta}{d \lambda}=2\left(\frac{n d}{\lambda^{3}}\right)^{1 / 2}
$$

The resolution at $\theta=0$ is given by [6]

$$
A=\frac{2 n d F}{\lambda}
$$

where $F$ is the finesse of the etalon.

The number of pixels per unit wavelength is then

$$
\frac{d N}{d \lambda}=\frac{2 n d F}{\lambda^{2}} \text {. }
$$

However, the etalon is only useful within one free spectral range (13). The total number of pixels is then

$$
N=\frac{d N}{d \lambda} \Delta \lambda=F
$$

which is usually of the order of 100 . Similarly, the total useful deflection angle is given by (12).

The last dispersive element to be discussed is the phasematched frequency doubler. If the fundamental beam is well focused inside a frequency doubler crystal there exists a solid angle within which sufficient fundamental beam power is available. The second harmonic beam will then form along the direction where phase matching occurs within a cone with an angle of apex given by [7]

$$
\Delta \theta=\frac{0.44 \lambda / l}{\partial\left(n_{1}-n_{2}\right) / \partial \theta}
$$

where $l$ is the crystal length and

$$
\frac{\partial\left(n_{1}-n_{2}\right)}{\partial \theta} \simeq\left(n_{2}^{o}-n_{2}^{e}\right) \sin 2 \theta_{0} .
$$

$n_{2}^{o}$ and $n_{2}^{e}$ are refractive indexes of ordinary and extraordinary waves at the second harmonic frequency and $\theta_{0}$ is the phasematch angle (between optic axis and propagation direction). Only the wavelength interval $\Delta \lambda$ contributes to the second harmonic beam [7]

$$
\Delta \lambda=\frac{0.44 \lambda / l}{\partial n_{1} / \partial \lambda-\frac{1}{2} \partial n_{2} / \partial \lambda_{2}}
$$

where

$$
\frac{\partial n_{1}}{\partial \lambda}-\frac{1}{2} \frac{\partial n_{2}}{\partial \lambda_{2}}=\frac{\partial n_{1}}{\partial \lambda}-\frac{1}{2}\left(\cos ^{2} \theta_{0} \frac{\partial n_{2}^{o}}{\partial \lambda}+\sin ^{2} \theta_{0} \frac{\partial n_{2}^{e}}{\partial \lambda}\right)
$$

and $n_{1}$ is the refractive index for the fundamental beam.

If the wavelength of the fundamental beam is changed a little, the direction of phase match is also changed so that

$$
\frac{d \theta}{d \lambda}=\frac{\partial n_{1} / \partial \lambda-\frac{1}{2} \partial n_{2} / \partial \lambda_{2}}{\partial\left(n_{1}-n_{2}\right) / \partial \theta} .
$$

The second harmonic beam is thus deflected according to (22) by varying the wavelength of the fundamental beam. Since two beams can just be resolved when they are $\frac{1}{2} \Delta \theta$ or $\frac{1}{2} \Delta \lambda$ apart, the number of pixels per unit wavelength is 
TABLE I

Properties of Different Dispersive Beam Deflectors Computed from (5)-(26). $d \theta / d \lambda$ and $d N / d \lambda$ are the Deflection Angle and Number of Pixels per Unit Wavelength, $N$ is the Total Number of Pixel.s Determined by the Tuning Range (120 nm). $\Delta \theta$ and $\Delta \lambda$ are Angle and Wavelength Intervals per Pixel. The Parameters Used are for the Prism: $b=50 \mathrm{~mm}$, $h=43.3 \mathrm{~mm}, d n / d \lambda=4 \cdot 10^{-5} \mathrm{~nm}^{-1}$; FOR THE GRATING: $d=1 \mu \mathrm{m}, m=1, \theta_{o}=0, D=20 \mathrm{~mm}$; FOR THE FABRY-PEROT: $n d=50 \mathrm{~mm}$, $F=100 ;$ AND FOR THE FREQuency Doubler $\left(\mathrm{LiIO}_{3}\right): l=14 \mathrm{~mm}, \delta=5.3 \mathrm{~mm}, \theta_{0}=45^{\circ}, n_{1}=n_{2}=1.870, \partial n_{1} / \partial \lambda=-0.0683 \mu \mathrm{m}^{-1}$, $\partial n_{2}^{o} / \partial \lambda=-0.566 \mu \mathrm{m}^{-1}, \partial n_{2}^{e} / \partial \lambda=-0.529 \mu \mathrm{m}^{-1}, n_{2}^{o}=1.96, n_{2}^{e}=1.79$. (With $\delta=5.3 \mathrm{~mm}$ (23) AND (26) YIELD the SAME Results.) Everywhere the Wavelength $750 \mathrm{~nm}$ Has BeEn Used, a Tuning Range of $120 \mathrm{~nm}$ Has Been Assumed Corresponding to the Parameters of the Alexandrite Laser.

\begin{tabular}{|l|c|c|c|c|c|}
\hline & $\frac{\mathrm{d} \theta}{\mathrm{d} \lambda}$ & $\frac{\mathrm{dN}}{\mathrm{d} \lambda}$ & $\mathrm{N}$ & $\Delta \theta$ & $\Delta \lambda$ \\
\hline & $\mathrm{mrad} / \mathrm{nm}$ & $\mathrm{pixels} / \mathrm{nm}$ & $\mathrm{pixels}$ & $\mathrm{mrad}$ & $\mathrm{nm}$ \\
\hline PRISM & 0.05 & 3.2 & 350 & 0.016 & 0.31 \\
\hline GRATING & 1.5 & 27 & 3200 & 0.056 & 0.038 \\
\hline FABRY-PEROT & 890 & $2.5 \times 10^{4}$ & 100 & 0.036 & $4.0 \times 10^{-5}$ \\
\hline FREQ.DOUBLER & 1.2 & 17 & 2000 & 0.071 & 0.059 \\
\hline
\end{tabular}

$$
\frac{d N}{d \lambda}=\frac{1}{\frac{1}{2} \Delta \lambda}=\frac{\partial n_{1} / \partial \lambda-\frac{1}{2} \partial n_{2} / \partial \lambda_{2}}{0.22 \lambda / l}
$$

However, diffraction effects have not been considered here. The cone formed by the focused fundamental beam results in a beam diameter

$$
\delta=\frac{D}{f} \frac{l}{n_{1}}
$$

at the output face of the doubler crystal. Here $D$ is the diameter of the input laser beam, $f$ is the focal length of the focusing lens, $l$ is the crystal length, and $n_{1}$ is the refractive index for the fundamental beam. Since the second harmonic beam is formed within the diameter $\delta$, the diffraction angle is

$$
\Delta \theta_{D}=\frac{\lambda_{2}}{\delta}=\frac{f \lambda_{2} n_{1}}{D l}
$$

where $\lambda_{2}$ is the wavelength of the second harmonic light. The number of pixels per unit wavelength caused by the diffraction limit is then

$$
\left(\frac{d N}{d \lambda}\right)_{D}=\frac{d \theta}{d \lambda} \frac{1}{\Delta \theta_{D}}=\frac{D l}{f \lambda_{2} n_{1}} \frac{d \theta}{d \lambda} .
$$

In a practical design the parameters should be adjusted so that (23) and (26) yield the same number of pixels.

In Table $I$ actual numbers have been inserted into the expressions just derived. The table has been computed using the wavelength $750 \mathrm{~nm}$ and a tuning range of $120 \mathrm{~nm}$ corresponding to the parameters of the alexandrite laser. It is seen that the prism has a small angular dispersion, but a reasonable number of pixels. The grating yields a good angular deflection and a large number of pixels which can easily be increased by using larger gratings (larger spot sizes at the grating). The Fabry-Perot etalon has a very large angular dispersion, but it is only useful within one free spectral range of $0.004 \mathrm{~nm}(3 \mathrm{GHz})$ for the example shown in Table I where it yields a total deflection of $3.6 \mathrm{mrad}$ with about 100 pixels (number of pixels equals the finesse). Hence, the FabryPerot deflector can be applied in case of narrow bandwidth lasers like the usual gas lasers. The resonators of these must then be changed to include a tuning element which makes it possible to scan the output laser wavelength across the gain curve.

The phase-matched $\mathrm{LiIO}_{3}$ frequency doubler device shown in Table I has a performance similar to the grating. It has the advantage of being more compact and its output wavelength is UV where photosensitive materials usually are best when used with the alexandrite laser. Furthermore, the laser linewidth need not be so narrow as, for example, when the grating deflector is applied. We have investigated experimentally the $\mathrm{LiIO}_{3}$ deflector used as an example in Table I. We applied a fundamental beam with $\lambda=750 \mathrm{~nm}$ from a $\mathrm{CW}$ dye laser (with Oxazine dye) pumped by a krypton laser. The beam was focused with a $12.6 \mathrm{~mm}$ focal length lens into the $\mathrm{LiIO}_{3}$ crystal which was cut in a direction $45^{\circ}$ from the optic axis yielding type I phase matching for $750 \mathrm{~nm}$. The apex angle of the second harmonic beam was measured to be about 1 mrad (full angle measured outside the crystal). This is about a factor of 4 more than the $0.26 \mathrm{mrad}(0.071 \times 2 \times 1.87)$ mrad expected from Table I. However, $\delta$ [in (24)] was about $0.5 \mathrm{~mm}$ in the present experiment implying a full diffraction angle of $1.5 \mathrm{mrad}$. We measured a deflection rate of 4.3 $\mathrm{mrad} / \mathrm{nm}$ (measured outside the crystal). The internal deflection rate is a factor of $n=1.87$ less than the external one or $2.3 \mathrm{mrad} / \mathrm{nm}$ which is somewhat larger than predicted in Table I from (22). The total scan width obtained with the $12.6 \mathrm{~mm}$ focal length lens was 4 degrees (measured outside the crystal). This value agrees well with the focusing cone obtained with the $12.6 \mathrm{~mm}$ lens and input beam diameter of $1 \mathrm{~mm}$. The number of pixels in this setup was then about 140 , but can be increased by increasing the solid angle of the focusing cone of the fundamental beam.

As shown in Table I, a few thousand pixels can be obtained from both grating and doubler deflectors. With a maximum scan frequency of $5 \mathrm{MHz}$ [2] scan rates up to 10 gigapixels/s 
should be possible, making these deflectors considerably faster than usual acoustooptic deflectors which have maximum speeds of 20-30 megapixels/s [8]. A typical acoustooptic deflector scans some hundred pixels with a total deflection of the order of $10 \mathrm{mrad}$. Hence, some of the dispersive deflectors shown in Table $\mathbf{I}$ have better performance than the ordinary acoustooptic deflector.

\section{CONCLUSION}

It has been shown that it is possible to construct fast beam deflectors based on usual dispersive optical components by means of electrooptic tuning of broad-band lasers. Scans with several thousand pixels, about 10 degrees of deflection and with scanning rates up to 10 gigapixels/s can be obtained.

\section{REFERENCES}

[1] J. M. Telle and C. L. Tang, "New method for electrooptical tuning of tunable lasers," Appl. Phys. Lett., vol. 24, pp. 85-87, 1974.

[2] "Electro-scan tuner," Ithaca Research Corp., Ithaca, NY.

[3] M. Born and E. Wolf, Principles of Optics, fifth edition. Oxford, England: Pergamon, 1975 , p. 180.

[4] - Principles of Optics, fifth edition. Oxford, England: Pergamon, 1975 , p. 407.

[5] - Principles of Optics, fifth edition. Oxford, England: Pergamon, 1975, pp. 403-406.

[6] -, Principles of Optics, fifth edition. Oxford, England: Pergamon, 1975, pp. 330-335.

[7] W. F. Hagen and P. C. Magnante, "Efficient second-harmonic generation with diffraction-limited and high-spectral radiance Nd-glass lasers," J. Appl. Phys., vol. 40, pp. 219-224, 1969.

[8] B. R. Reddersen, "Highspeed acousto-optic scanning," Laser Focus, vol. 16 , pp. 54-61, Oct. 1980 .

\title{
Parametric Amplification and Frequency Conversion in Optical Fibers
}

\author{
ROGER H. STOLEN AND JOHN E. BJORKHOLM, SENIOR MEMBER, IEEE
}

(Invited Paper)

\begin{abstract}
We find that the parametric four-photon gain for light pulses decreases for fibers longer than a characteristic length. This length is related to the common experimental observation that stimulated parametric emission is usually prominent only in short fibers while in long fibers stimulated Raman scattering dominates. Despite the fact that the actual process involves an intensity dependent bandwidth and broadening of the pump linewidth from self-phase modulation, it is possible to develop a simple expression for the characteristic length which requires only the initial pump linewidth and the lowpower parametric bandwidth. This bandwidth can often be estimated from the pump wavelength and the measured frequency shif $t$ between the pump and the generated waves. Expressions for gain and amplification are derived from coupled wave equations and in the Appendixes it is shown that these are of the same form as the planewave equations, but modified by coupling coefficients called overlap integrals.
\end{abstract}

\section{INTRODUCTION}

A WIDE variety of nonlinear optical effects have been observed using optical fibers as the nonlinear medium. Stimulated Raman scattering and four-photon parametric mixing can be used to convert the input light into light at one or more different frequencies [1]. In multimode fibers,

Manuscript received January 7, 1982.

The authors are with Bell Laboratories, Holmdel, NJ 07733. both effects usually occur simultaneously although this need not necessarily be the case. In comparison with Raman frequency conversion, parametric frequency conversion is advantageous in that frequency up-conversion is possible as well as down-conversion, and the range of frequency shifts is broader. The same advantages apply when both processes are viewed as a means for amplifying light. A primary aim of this paper is to describe the circumstances which favor parametric output as opposed to Raman output. In particular, we treat the case of pulsed input light although much of the formalism is also applicable to the $\mathrm{CW}$ case.

The gain coefficient for four-photon parametric amplification is generally larger than that for stimulated Raman amplification. Consequently, the parametric output might be expected to always dominate over the Raman output. Using pulsed lasers, it has been observed experimentally that this is only true for fibers shorter than some characteristic length which we denote $l_{c}$. For fibers longer than $l_{c}$ the stimulated Raman output dominates. In this paper we show how $l_{c}$ can be evaluated and understood in terms of the frequency bandwidth of the parametric gain and self-phase modulation of the pulsed input light. We will only treat situations in which the shift between the frequencies of the generated light and the 